\title{
Drosophila Ogg1 is required to suppress 8-oxo-guanine accumulation following oxidative stress
}

\author{
Takashi Yasukawa, Yasuyuki Nakahara, Jun Hirai and Yoshihiro H. Inoue* \\ Insect Biomedical Research Center, Kyoto Institute of Technology, Matsugasaki, \\ Sakyo-ku, Kyoto 606-0962, Japan
}

(Received 5 November 2014, accepted 24 January 2015)

\begin{abstract}
Reactive oxygen species (ROS) generated during energy production processes are a major cause of oxidative DNA damage. A DNA glycosylase encoded by the Ogg1 gene removes oxidized guanine bases and is widely conserved. However, the biological role of the gene in individual organisms has not yet been characterized in Drosophila, which is a suitable model to study the influence of oxidative damage on senescence. Here, we performed a genetic analysis to confirm that Ogg1 plays an essential role in the removal of 8-oxo-guanines from nuclei. We first confirmed by quantitative real-time PCR that $\operatorname{Ogg} 1 \mathrm{mRNA}$ expression was reduced by $30-55 \%$ in $O g g 1$ mutants and in flies expressing inducible $O g g 1$ dsRNA compared to control flies. We then showed that additional accumulation of 8-oxo-guanines occurred in the nuclei of epithelial midgut cells after paraquat feeding in flies with downregulated Ogg1 expression. We confirmed that a transposon possessing the UAS sequence was integrated in the $5^{\prime}$-UTR of the Ogg1 alleles and that it is oriented in the same transcriptional direction as the gene. Using the Gal4/UAS system, which enables us to induce ectopic expression in Drosophila, we induced overexpression of $\operatorname{Ogg} 1$ by 40 -fold. We observed a lower amount of 8-oxo-guanine in the midgut epithelial cells of adults overexpressing Ogg1. These genetic data strongly suggest that the Drosophila Ogg1 ortholog CG1795 plays an essential role in the suppression of 8-oxo-guanines, consistent with its role in other organisms. Although adult flies with reduced Ogg1 expression failed to show elevated sensitivity to paraquat, those with Ogg1 overexpression showed resistance to oxidative stress by paraquat feeding and had a significantly longer lifespan in normal feeding conditions. These observations are consistent with the hypothesis that oxidative DNA damage by ROS accumulation is a major contributor to senescence.
\end{abstract}

Key words: 8-oxo-guanine, Drosophila, lifespan extension, Ogg1, oxidative stress

\section{INTRODUCTION}

Reactive oxygen species (ROS) induce damage in genomic as well as mitochondrial DNA (Lindahl, 1993; Wang et al., 1998; Cooke et al., 2003). The oxidized guanine base 8-oxo-guanine is one of the most common oxidative DNA lesions in a cell. 8-oxo-guanine forms mismatch pairs with adenine and produces G:C to T:A transversions during DNA replication (Moriya, 1993; Thomas et al., 1997). Therefore, the production of oxidized bases can lead to DNA mutagenesis, and the accumulation of such DNA damage eventually results in the development of many diseases and senescence (Barja,

Edited by Etsuko Matsuurai

* Corresponding author. E-mail: yhinoue@kit.ac.jp
2004; Maynard et al., 2009; Kryston et al., 2011). To prevent these effects, oxidized DNA bases are initially removed by the base-excision repair mechanism (Wallace, 1998; Hazra and Mitra, 2006; Nemec et al., 2010). First discovered in Escherichia coli, the DNA glycosylase MutM excises 8-oxo-guanines generated by ROS (Michaels et al., 1992; Grollman and Moriya, 1993; Boiteux and Radicella, 1999). In Saccharomyces cerevisiae, a DNA glycosylase encoded by the $\operatorname{Ogg} 1$ gene catalyzes the removal of 8-oxoguanines and formamidopyrimidines from oxidatively damaged DNA (Nash et al., 1996; van der Kemp et al., 1996; Girard et al., 1997). Mammalian cells also express an ortholog of Ogg1 that plays an important role in the prevention of mutagenesis and carcinogenesis (Aburatani et al., 1997; Arai et al., 1997; Lu et al., 1997; Radicella et al., 1997; Rosenquist et al., 1997; Chevillard et al., 1998; 
Sakumi et al., 2003). It was shown that the Ogg1 ortholog in human cells is a major enzyme to repair 8-oxoguanine DNA lesions (Monden et al., 1999; Boiteux and Radicella, 2000).

To understand the biological function of $\operatorname{Ogg} 1$ in individual organisms rather than in cultured cells, knockout mouse lines carrying the null allele generated by gene disruption have been established (Klungland et al., 1999; Minowa et al., 2000). Ogg1-deficient mice are viable, but during aging they accumulate up to $>7$-fold more 8-oxoguanines than wild-type mice. In addition to biochemical evidence indicating that the human OGG1 gene plays an important role in removing 8-oxo-guanines, OGG1 is a strong candidate gene for susceptibility to human cancers and metabolite-related diseases (Audebert et al., 2000; Mao et al., 2007; Nakabeppu et al., 2007; Paz-Elizur et al., 2008; Okasaka et al., 2009; Sampath et al., 2012). However, genetic analyses that verify the relationship between $\operatorname{Ogg} 1$ expression and diseases using animal models remain challenging tasks.

Drosophila melanogaster has been the subject of intensive genetic analyses investigating the role of genes implicated in various biological phenomena (Pandey and Nichols, 2011; Prüßing et al., 2013). Drosophila also possesses an Ogg1 ortholog, which excises 8-oxo-guanines from $\gamma$-irradiated DNA in vitro. It is expressed at a low level in proliferative cells (Dherin et al., 2000). The Ogg1/CG1795 gene was also identified from a genomewide RNAi screen as a candidate gene involved in sensitivity to methyl methanosulfonate-induced DNA damage (Ravi et al., 2009). However, because neither genetic nor developmental analyses using Ogg1 mutants or RNAi flies have yet been performed (see Flybase, http://flybase. org), it is unclear whether the enzyme plays an important role in the DNA repair of oxidative damage after the incorporation of exogenous oxidants or via the ROS produced during intracellular metabolic processes.

In this study, we first confirmed that $O g g 1$ gene expression was down-regulated by $30-55 \%$ in transposoninduced mutants as well as in Drosophila adults expressing Ogg1 dsRNA. Accumulation of 8-oxo-guanine was observed in the nuclei of epithelial midgut cells of flies fed with paraquat, which produces free radicals within a cell and thus causes oxidative stress (Cochemé and Murphy, 2008). Conversely, when we induced $\sim 40$-fold overexpression of Ogg 1 in adults using the Gal4/UAS system, we observed significantly less accumulation of 8-oxoguanine. A positive correlation between Ogg1 expression and the extent of 8-oxo-guanine accumulation strongly suggested that the Ogg1 ortholog encoded by CG1795 plays an important role in oxidized base accumulation in Drosophila. Interestingly, flies overexpressing Ogg1 had significantly extended adult lifespans and showed resistance to oxidative stress. Our genetic data suggest that these Drosophila mutants and flies with modified $\operatorname{Ogg} 1$ gene expression offer promising models to clarify the relationship between DNA oxidation and senescence or the onset of several diseases.

\section{MATERIALS AND METHODS}

Drosophila stocks All D. melanogaster stocks were maintained on standard food at $25^{\circ} \mathrm{C}$. To express $O g g 1$ dsRNA, $P\left\{U A S-O g g 1 R N A i^{H M C 03426}\right\}$ (UAS-Ogg1RNAi $i^{H M C 03426}$ ) and P\{UAS-Ogg1RNAi $\left.i^{k k 101291}\right\}$ (UAS-Ogg1RNAi $\left.i^{k k 101291}\right)$ stocks were used. The former RNAi stock was obtained from the Bloomington Drosophila Stock Center. The latter was provided by the Vienna Drosophila RNAi Center. P\{Actin5C-Gal4\}25F01 (Actin5C-Gal4) was obtained from the Bloomington Drosophila Stock Center and used as a Gal4 driver. $P B\{W H\} O g g 1^{f 01384}\left(O g g 1^{f 01384}\right)$ and $P B\{W H\} O g g 1^{f 08013}\left(O g g 1^{f 08013}\right)$ were obtained from the Harvard Medical School Exelixis Collection. Before analyzing individual flies with modified Ogg1 expression in an adult lifespan assay, each stock was outcrossed into the $w^{1118}$ background for at least three generations. Control flies (Actin5C-Gal4/+, UAS-Ogg1RNAi ${ }^{H M S 03426} /+$, $\left.U A S-O g g 1 R N A i^{k k 101291} /+\right)$ were generated from each outcross to $w^{1118}$. Flies depleted of Ogg 1 mRNA were obtained from a cross between Actin5C-Gal4 and UASOgg $1 R N A i^{H M S 03426}$ or UAS-Ogg1RNAi $i^{k k 101291}$. Males overexpressing Ogg1 were generated from an outcross between Ogg1 mutant females and Actin5C-Gal4 males.

Lifespan assay For lifespan analysis, adult male flies were collected within $24 \mathrm{~h}$ after eclosion; 20 flies were reared in a single plastic vial on Drosophila instant medium (Formula 4-24, Blue, Biological Supply Company, Burlington, NC, USA) at $25^{\circ} \mathrm{C}$ or $28^{\circ} \mathrm{C}$ for induced gene expression dependent on the Gal4/UAS system. Dead adults were scored every day and food vials were changed every three days.

For the paraquat feeding assay, Ogg 1 mutant flies and flies depleted of or overexpressing Ogg 1 mRNA were reared on instant medium containing $10 \mathrm{mM}$ paraquat (methyl viologen dichloride hydrate (Sigma-Aldrich, St. Louis, MO, USA)). Survival curves were analyzed by the Kaplan-Meier procedure. Significance was determined by the log-rank test.

Quantitative real-time PCR analysis qRT-PCR analysis was performed to determine the expression level of Ogg 1 genes in adult flies with induced expression of each dsRNA or of Ogg1 with a Gal4 driver. Total RNA was extracted from adult flies with each genotype using the TRIzol reagent (Invitrogen, Carlsbad, CA, USA). cDNA synthesis from the total RNA was carried out using a PrimeScript II High Fidelity RT-PCR kit (Takara, Shiga, Japan) with random primers. qRT-PCR was performed using FastStart Essential DNA Green Master (Roche, 
Mannheim, Germany) and a LightCycler Nano (Roche, Basel, Switzerland). The following primers were used for amplification: FW, 5'-TGCGTATACATTTCCCACGA3'; RV, 5'-GCTATGAACTTGGCCCGATA-3'. RP49 was used as a normalization reference (Oka et al., 2015). Relative mRNA levels were quantified using LightCycler Nano software version 1.0 (Roche, Basel, Switzerland).

8-oxo-guanine immunostaining procedures and evaluation Anti-8-oxo-guanine immunostaining of adult midguts was largely performed as described by Park et al. (2012). We observed the surface layers of epithelial lumen cells in the posterior region using a confocal microscope. Three-day-old adult males were kept for $16 \mathrm{~h}$ on instant medium containing $10 \mathrm{mM}$ paraquat after a 2 -h starvation period. Midguts dissected from the adults were treated with $20 \mu \mathrm{g} / \mathrm{ml}$ DNase-free RNase (Nippongene, Tokyo, Japan) for $30 \mathrm{~min}$ at $37{ }^{\circ} \mathrm{C}$ as described in Yamaguchi et al. (2006). Subsequently, they were fixed with Carnoy's solution and then incubated in $2 \mathrm{~N} \mathrm{HCl}$ for 2 min. After successive washing, the fixed samples were incubated with anti-8-oxo-guanine antibody (Japan Institute for the Control of Aging, Fukuroi, Japan) as a primary antibody and then with anti-mouse IgG conjugated with Alexa594 (Invitrogen, Carlsbad, CA, USA), and mounted with Vectashield (Vector Laboratories, Burlingame, CA, USA). Samples were observed with an Olympus laser scanning confocal microscope (Fv10i, Olympus, Tokyo, Japan). Brightness and contrast of entire images were adjusted using FV10i software. For the quantitative analysis of 8-oxo-guanine-positive cells, images were processed by Photoshop. The numbers of epithelial cells were counted in a $210 \times 210 \mu \mathrm{m}^{2}$ region of the posterior midgut and cells positive for anti-8-oxo-guanine immunostaining in the images were judged using ImageJ software (http://rsbweb.nih.gov/ij/download.html). For each genotype, midguts from more than 10 individual flies were examined and at least 1,000 nuclei were observed in total.

Statistical analysis Statistical analyses were performed with Excel (Microsoft, Redmond, WA, USA) and p-values were calculated with Student's $t$-tests or the log-rank test.

\section{RESULTS AND DISCUSSION}

Reduced expression of Drosophila Ogg1 in transposon-induced mutants and in flies depleted of Ogg1 mRNA by induced dsRNA expression To examine whether the $D$. melanogaster gene CG1795, encoding an Ogg1 ortholog, is required for the removal of oxidized guanine bases in the DNA repair process, we examined the accumulation of 8-oxo-guanines in transposon-induced mutants of the $\operatorname{Ogg} 1$ gene. For the two alleles, $\operatorname{Ogg} 1^{f 01384}$ and $\operatorname{Ogg} 1^{f 08013}$, the PiggyBac transposon $P B a c\{w[+m C]=$
$W H\}(P B a c\{W H\})$ was inserted into the 5'-UTR sequences (Thibault et al., 2004). The Ogg $1^{f 08013}$ insertion was integrated $277 \mathrm{bp}$ upstream of the ATG codon (see Fig. 1A; http://flybase.org). The insertion in $O g g 1^{f 01384}$ was integrated $13 \mathrm{bp}$ upstream of $\operatorname{Ogg} 1^{f 08013}$. We determined the orientation of these insertions by PCR using the DNA sequences of the $\operatorname{Ogg} 1$ gene and $P B a c\{W H\}$ as primers. Both insertions were integrated within the gene in the same transcriptional orientation as the UAS sequences (Fig. 1A). Our quantitative real-time PCR (qRT-PCR) analysis revealed that $\operatorname{Ogg} 1 \mathrm{mRNA}$ expression was less than $60 \%$ of the level observed in the controls for both insertional mutants (Fig. 1B). Next, we further examined whether the $O g g 1 \mathrm{mRNA}$ was efficiently depleted by ubiquitous expression of dsRNA comprising $O g g 1$ mRNA and its complement. Induced expression of two different dsRNA sequences using UAS-Ogg1RNAi $i^{H M C 03426}$ and UAS-Ogg1RNAi $i^{k k 101291}$ was carried out. The Ogg1 mRNA expression level was reduced to $45.3 \%$ and $70.3 \%$, respectively, relative to the normal control level (Actin5CGal4/+, Fig. 1C). Ogg1 mRNA was successfully depleted by induced expression of these dsRNAs.

Adult flies with reduced Ogg1 mRNA exhibit hyperaccumulation of 8-oxo-guanines in midgut cells after paraquat feeding Having confirmed a decrease in Ogg1 mRNA in the Ogg1 mutants as well as in flies depleted of $\operatorname{Ogg} 1 \mathrm{mRNA}$, we next examined whether the number of epithelial cells containing oxidized DNA bases increased in adult midguts after paraquat feeding. We examined the presence of 8-oxo-guanines in posterior midgut cells from adult males after $16 \mathrm{~h}$ of feeding on diet containing $10 \mathrm{mM}$ paraquat, because the midgut epithelial cells are exposed earlier to chemicals contained in the diet and continue direct contact with them for a long time. We selected adult males that ingested paraquat based on the presence of a blue dye contained in the diet as a marker.

After anti-8-oxo-guanine immunostaining of the adult midguts, we observed a layer of epithelial lumen cells at the posterior region of the midgut using a confocal microscope and examined at least 1,200 midgut epithelial cells from more than 10 individual flies (Fig. 2). In control flies $\left(w^{1118}\right), 38.6 \%$ of 2,884 midgut epithelial cells $(\mathrm{n}=14$ flies) in total were judged as 8-oxo-guanine-positive (Fig. 2, A and E). On the other hand, we scored positive cells more frequently $(60.4 \%$ of 1,782 cells $)$ in epithelial cells from Ogg $1^{f 01384}$ mutant males ( $\mathrm{n}=11$ flies) (Fig. 2, B and E). The frequency in the mutants was significantly higher than the control flies $(\mathrm{p}<0.01)$. A statistically significant increase in the frequency of positive cells $(58.3 \%$ of 2,226 cells, $\mathrm{n}=13$ flies) was also found in the $\operatorname{Ogg} 1^{f 08013}$ mutant males $(\mathrm{p}<0.01)$. In Ogg1 mRNA-depleted male flies (Actin5C-Gal4/UAS-Ogg1RNAi ${ }^{H M C 03426}$ (Fig. 2, D and F) and Actin5C-Gal4/UAS-Ogg1RNAi $i^{k k 101291}$ (Fig. 2F)), 
A
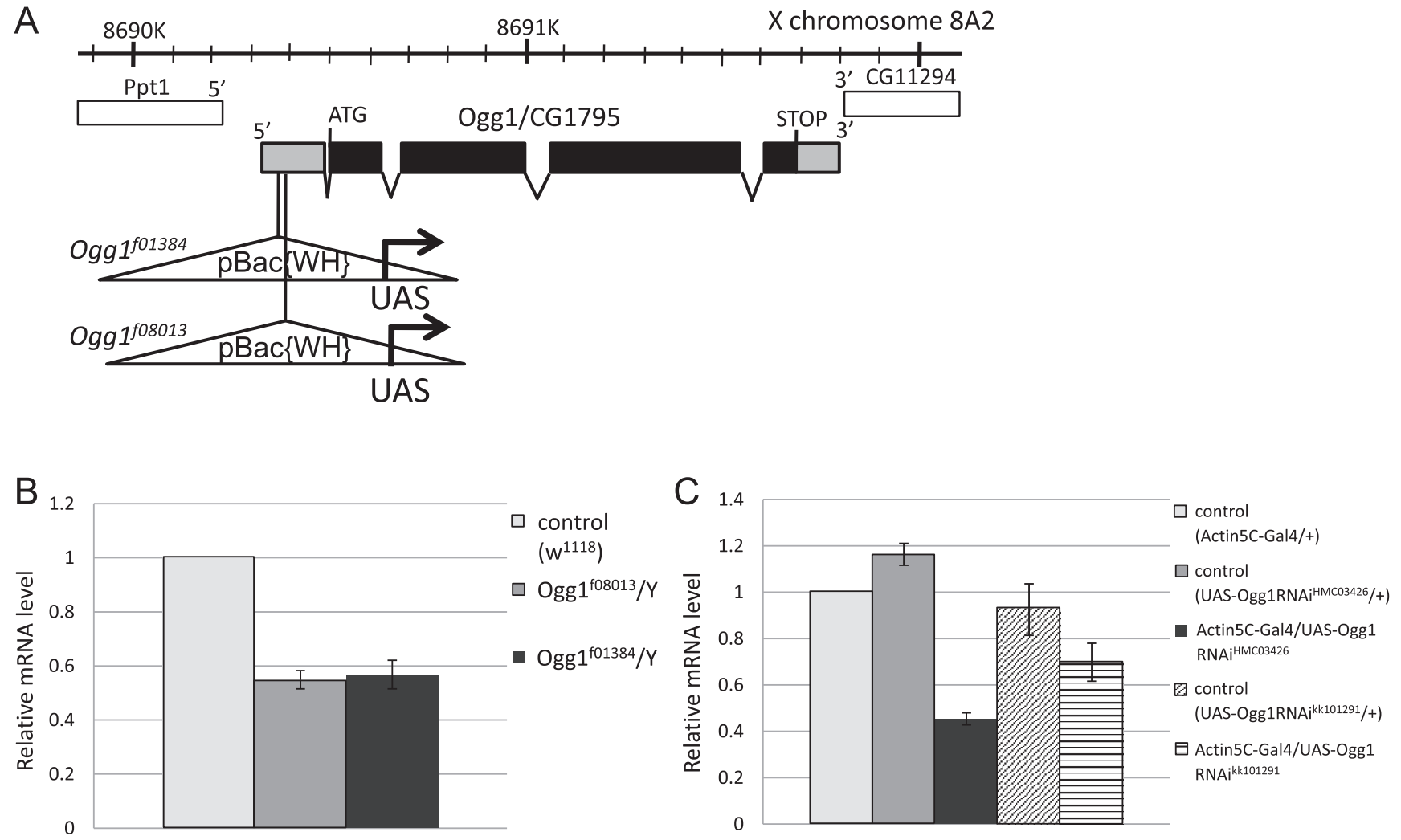

Fig. 1. Insertional mutations of a gene encoding Drosophila Ogg1 ortholog and reduction of Ogg1 mRNA in mutants and in flies expressing Ogg1 dsRNAs. (A) Genomic map of two $p B a c\{W H\}$ insertional mutations in the Ogg1 locus. Gray bars represent the 5'or 3'-UTR of Ogg1. Black bars are coding exons. Open bars show the surrounding genes. Both insertions carrying UAS sequences are integrated into the $5^{\prime}$-UTR region of the gene in the same orientation with respect to Ogg1 transcription. (B) Relative mRNA level of $O g g 1$ mRNA from adult males of two insertional mutants $\left(O g g 1^{f 01384} / Y\right.$ and $\left.O g g 1^{f 08013} / Y\right)$ was calculated on the basis of real-time qRT-PCR analysis. (C) Relative mRNA level of Ogg1 mRNA from males inducibly expressing dsRNAs for Ogg1 (Actin5C-Gal4/UASOgg1RNAi $i^{H M C 3426}$ and Actin5C-Gal4/UAS-Ogg1RNAi $i^{k k 101291}$ ) was calculated on the basis of real-time qRT-PCR analysis. Data are represented as averages of three independent experiments and error bars represent the standard deviation.

the 8-oxo-guanine-positive cells were also scored more frequently ( $65.5 \%$ of 3,346 cells $(\mathrm{n}=18$ flies) and $70.7 \%$ of 3,762 cells $(\mathrm{n}=16$ flies), respectively), compared to the controls (Actin5C-Gal4/ $+(40.3 \%$ of 1,269 cells, $\mathrm{n}=10$, Fig. $2 \mathrm{~F}$ ), UAS-Ogg1RNAi ${ }^{H M C 03426}$ (31.2\% of 1,934 cells, $\mathrm{n}=$ 11 flies, Fig. 2, C and F), UAS-Ogg1RNAik101291 (38.5\% of $1,549$ cells, $\mathrm{n}=10$ flies, Fig. $2 \mathrm{~F})$ ). The $\mathrm{p}$-value indicating statistical significance was less than 0.01 in every case. These genetic data indicate that reduced expression of the Ogg1 gene resulted in additional accumulation of 8-oxo-guanines in midgut epithelial cells under oxidative stress.

Adult flies with reduced $O g g 1$ expression fail to show increased sensitivity to paraquat Next, we investigated whether adult flies with reduced $\operatorname{Ogg} 1$ expression and additional accumulation of 8-oxo-guanines exhibit hypersensitivity to paraquat. We selected the flies (Actin5C-Gal4/UAS-Ogg1RNAi ${ }^{H M C 03426}$ ) in which Ogg1 mRNA was depleted most efficiently and performed a paraquat sensitivity assay to examine the survival rate of male flies fed on a diet supplemented with $10 \mathrm{mM}$ paraquat. As shown in Fig. 3, the viability curve of Ogg1-depleted male flies was indistinguishable from that of the controls (Actin5C-Gal4/+ and UASOgg 1RNAi $\left.i^{H M C 03426} /+\right)$ under oxidative stress. Both Ogg1 mutants, $O g g 1^{f 01384}$ and $\operatorname{Ogg} 1^{f 08013}$, showed a longer lifespan than the $O g g 1$ mRNA-depleted flies or the controls (data not shown). Neither the Ogg1 mutants nor the Ogg1 mRNA-depleted flies showed hypersensitivity to paraquat. The $30-55 \%$ reduction of $O g g 1$ expression may have been insufficient for a shift to paraquat hypersensitivity. Alternatively, it has been reported that ribosomal protein $\mathrm{S} 3$ (dRpS3) also has 8-oxo-guanine repair activity (Yacoub et al., 1996; Cappelli et al., 2003). The dRpS3 activity may be sufficient to repair the 8-oxo-guanine in midguts with reduced Ogg1 expression. It will be of interest to examine genetic interaction between $O g g 1$ and $d R p S 3$ in the repair of oxidative DNA damage in a future study. 

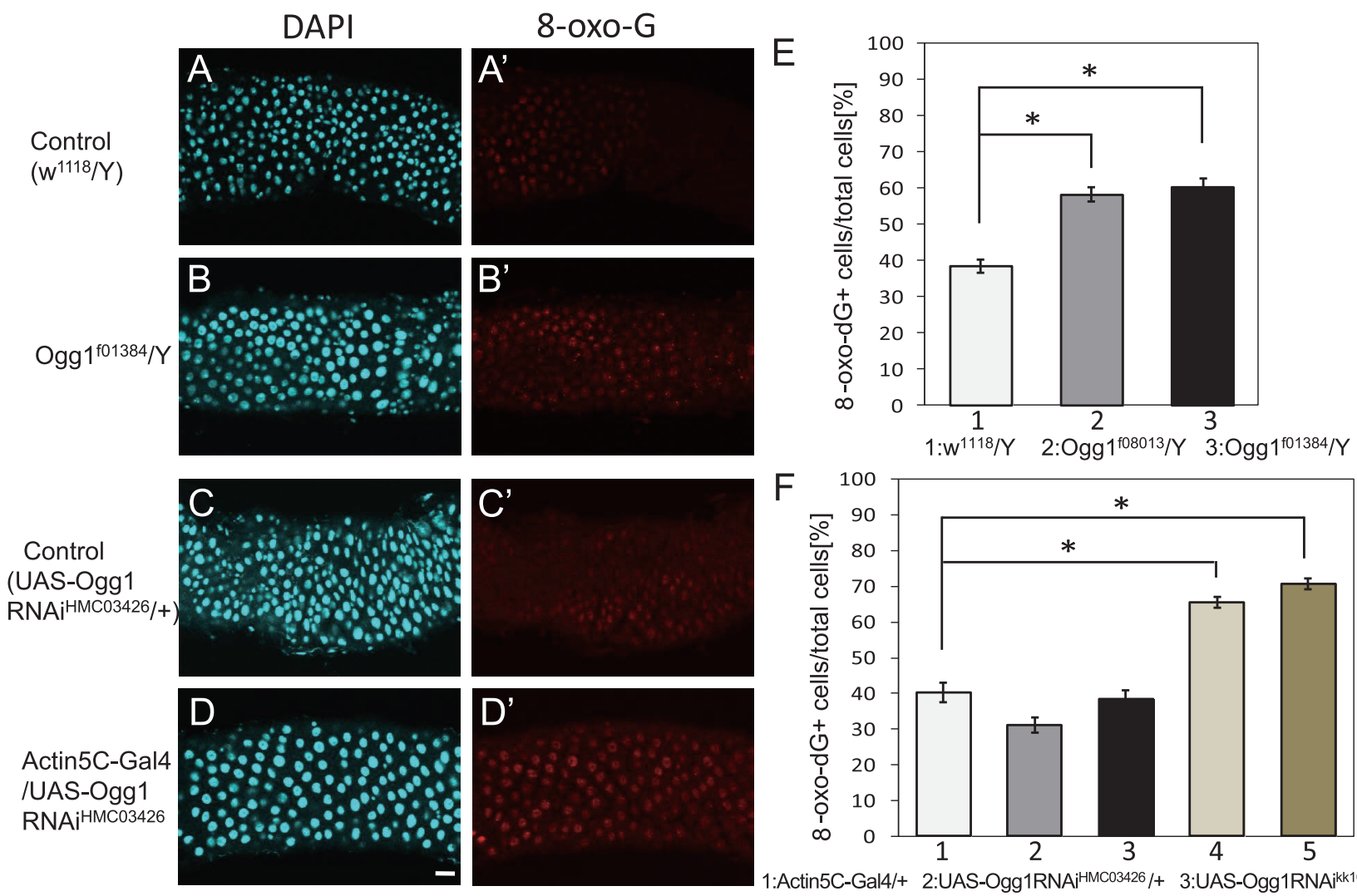

1:Actin5C-Gal4/+ 2:UAS-Ogg1RNAiHMC03426/+ 3:UAS-Ogg1RNAikk101291/+ 4:Actin5C-Gal4/UAS-Ogg1RNAiHMC03426 5:Actin5C-Gal4/UAS-Ogg1RNAikk101291

Fig. 2. Immunodetection of 8-oxo-guanines accumulated in posterior midgut cells of $O g g 1$ mutants and adults with depleted $O g g 1$ mRNA after paraquat feeding. (A)-(D) Typical confocal images of epithelial cells from the posterior midgut region immunostained with anti-8-oxo-guanine antibody in Ogg1 mutant adult males and adult males with depleted Ogg1 mRNA after $16 \mathrm{~h}$ of feeding on food containing $10 \mathrm{mM}$ paraquat. (A, A') Control epithelial midgut cells from a $w^{1118}$ male, stained with DAPI (A) or anti-8-oxo-guanine antibody (A'). (B, B') Midgut cells from an $O g g 1^{f 01384} / \mathrm{Y}$ fly, stained with DAPI (B) or antibody (B'). (C, C') Control midgut cells from a UAS-Ogg1RNAi ${ }^{H M C 03426} /+$ adult, stained with DAPI (C) or antibody (C'). (D, D') Ogg1 mRNA-depleted midgut cells from an Actin5CGal4/UAS-Ogg1RNAi ${ }^{H M C 03426}$ adult, stained with DAPI (D) or antibody (D'). (E, F) Frequencies of epithelial midgut cells stained with the antibody after paraquat feeding. Midgut cells having 8-oxo-guanine accumulation in their nuclei significantly increased in the Ogg1 mutants (E) and in the Ogg1 mRNA-depleted adults (F). Error bars represent the standard deviation. *, p < 0.01 (Student's $t$-test). Scale bar, $10 \mu \mathrm{m}$.

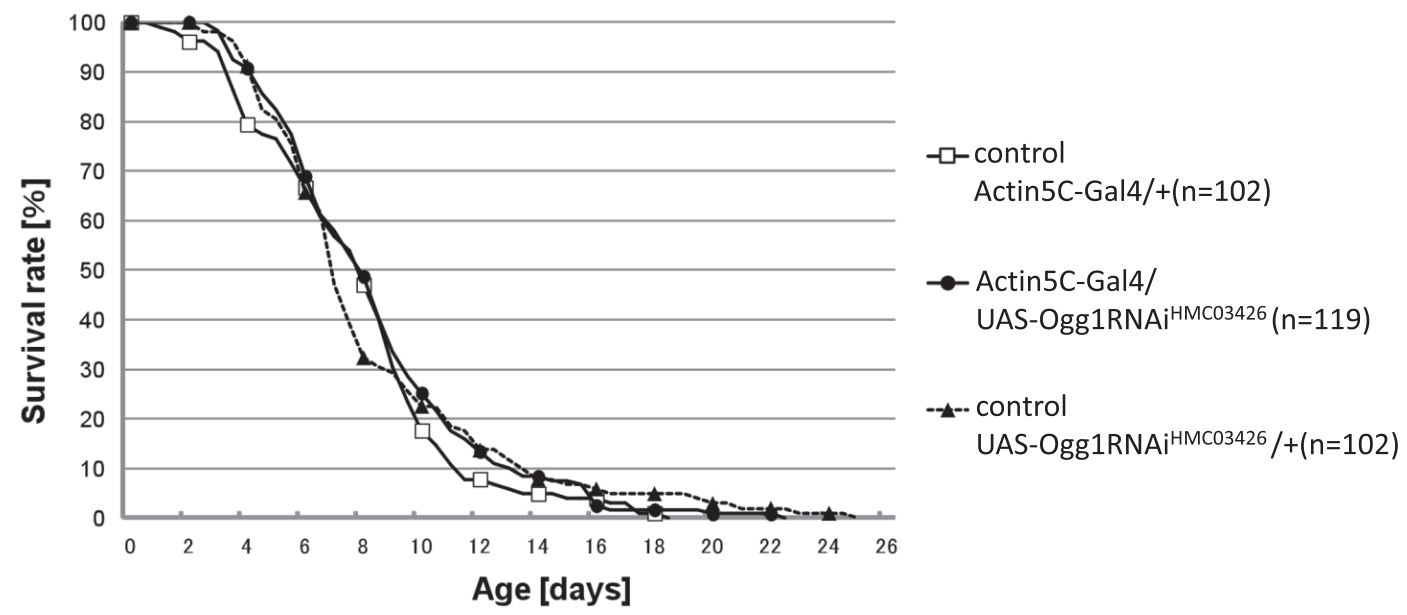

Fig. 3. Paraquat sensitivity assays of Ogg1 mutant adults and adults with depleted Ogg 1 mRNA. Lifespan curves of adult males depleted of Ogg1 mRNA (Actin5C-Gal4/UAS-Ogg1RNAi ${ }^{H M C 03426}$ ), and two control males (Actin5C-Gal4/+ and UAS$\left.\operatorname{Ogg} 1 R N A i^{H M C 03426} /+\right)$. These adults were collected within $24 \mathrm{~h}$ after eclosion and reared on a diet containing $10 \mathrm{mM}$ paraquat immediately after they were collected. 
Overexpression of Drosophila Ogg1 using $P B a c\{W H\}$ insertions possessing UAS sequences and a Gal4 driver As described above, we showed by PCR analysis that PBac $\{W H\}$ insertions in $O g g 1^{f 01384}$ and Ogg $1^{f 08013}$ mutants were integrated into the 5'-UTR of the gene in the same transcriptional orientation as the UAS. Because PBac $\{W H\}$ possesses 14 repeats of the UAS sequence, these two alleles may allow us to induce overexpression dependent on the Gal4 transcription factor. In fact, we confirmed a 36-fold increase of Ogg1 mRNA in Ogg $1^{f 01384} / Y$;Actin5C-Gal4/+ adult flies by qRT-PCR, compared with the control. We also observed a 39-fold increase in Ogg1 mRNA in adult $O g g 1^{f 08013} /$ Y;Actin5C-Gal4/ + flies (Fig. 4). We have failed to detect Drosophila Ogg1 ortholog polypeptide in western blot experiments using three independent Ogg1 antibodies against the human OGG1 ortholog (orb5706 (Biorbyt, Cambridge, UK), ab135940 (Abcam, Cambridge, UK), SC376935 (Santa Cruz Biotechnology, Dallas, TX, USA)). It will be important to confirm the modified gene expression of Ogg1 described here at the protein level in our future study, and production of a specific antibody against the Drosophila Ogg1 ortholog is currently underway.

Ogg1 overexpression decreases accumulation of 8oxo-guanines in Drosophila midgut cell nuclei after paraquat feeding Next, we further examined whether nuclear accumulation of 8-oxo-guanines is suppressed in adults overexpressing Ogg1. As described above, we carried out anti-8-oxo-guanine immunostaining of adult midguts for controls and flies with $\operatorname{Ogg} 1$ overex-

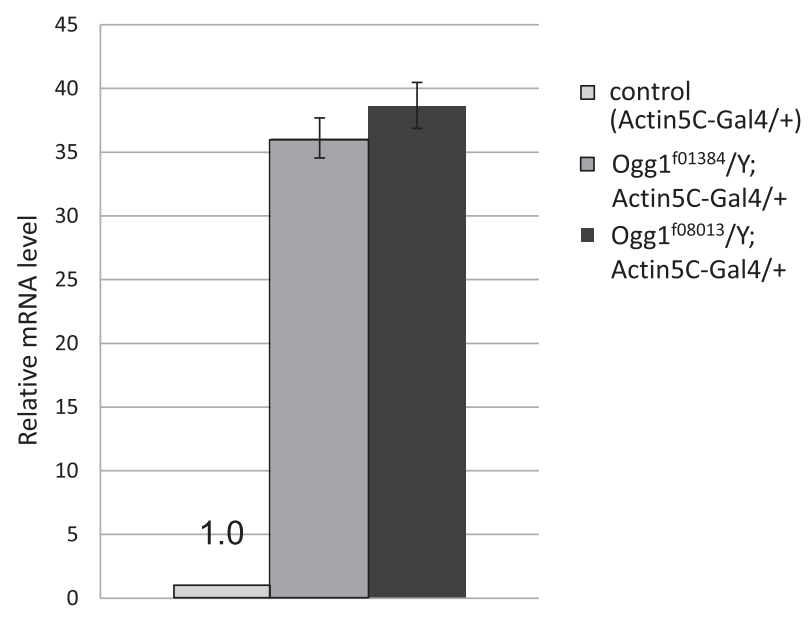

Fig. 4. Overexpression of Ogg1 mRNA in mutants carrying $P B\{W H\}$ insertions that possess UAS sequences and Actin5CGal4. qRT-PCR experiments were carried out to quantify $O g g 1$ mRNA expression in adult males $\left(O g g 1^{f 01384} / Y\right.$;Actin5C-Gal4 / + and $O g g 1^{\text {fo8013 }} /$ Y;Actin5C-Gal4/+). Overexpression of 36- and 39-fold was observed in these adult males, compared with the controls carrying Actin5C-Gal4 alone. Data are represented as averages of three independent experiments and error bars represent the standard deviation. pression (Ogg1 $1^{f 01384} / Y$;Actin5C-Gal4/+ and Ogg1 $1^{f 08013} /$ $Y$;Actin5C-Gal4/+) after $10 \mathrm{mM}$ paraquat feeding (Fig. 5). In control flies (Actin5C-Gal4/+, Fig. 5, A and D), 8oxo-guanine-positive cells were observed in $40.3 \%$ of total epithelial cells examined (1,269 cells, $\mathrm{n}=10$ flies). However, in $O g g 1^{f 01384} / Y$;Actin5C-Gal4/ + flies, positive cells comprised only $13.3 \%$ of the total epithelial midgut cells (1,729 cells, $\mathrm{n}=10$ flies) (Fig. $5, \mathrm{~B}$ and $\mathrm{D}$ ). The same result was also obtained in $O g g 1^{f 08013} / Y$;Actin5C-Gal4/+ flies (Fig. 5, C and D), in which 8-oxo-guanine-positive cells appeared less frequently $(13.2 \%)$ in the total epithelial cells examined ( 1,633 cells, $\mathrm{n}=10$ flies $)$. In both cases, 8-oxo-guanine accumulation in midgut epithelial cells was significantly lower than with Actin5C-Gal4/+ $(\mathrm{p}<0.01)$ as well as with $O g g 1^{f 01384} / Y$ and $O g g 1^{f 08013} / Y$ $(\mathrm{p}<0.01)$. These genetic data indicated that Ogg1 overexpression suppresses the accumulation of 8-oxoguanines in Drosophila adult midgut cells after paraquat feeding. Taken together with data from adults with reduced Ogg1 expression, these results lead us to conclude that CG1795 encoding Drosophila Ogg1 has an essential role in the suppression of oxidized guanines generated by oxidative stress, as reported in $E$. coli, budding yeast and mice (Michaels et al., 1992; Grollman and Moriya, 1993; Nash et al., 1996; van der Kemp et al., 1996; Girard et al., 1997; Klungland et al., 1999; Minowa et al., 2000).

Adult flies with Ogg1 overexpression show significantly extended lifespan and resistance to paraquat We further examined whether overexpression of the Drosophila Ogg1 ortholog would lead to adult lifespan extension in normal feeding conditions, i.e., without paraquat. Interestingly, Ogg $1^{f 01384} / Y$;Actin5C-Gal4/+ adult males with 36 -fold higher $O g g 1$ expression showed a significantly extended lifespan, compared with Ogg $1^{f 01384} / Y$ males $(\mathrm{p}<0.01$, log-rank test) and with Actin5C-Gal4 $/+$ males ( $\mathrm{p}<0.01$, log-rank test) (Fig. $6 \mathrm{~A})$. We obtained essentially the same results in experiments using another $P B\{W H\}$ insertion allele, $O g g 1^{f 08013} /$ $Y$ (Fig. 6B). The Ogg $1^{f 08013} / Y$;Actin5C-Gal4/+ adult males with 39-fold overexpression of Ogg1 showed a significantly extended lifespan, compared with $O g g 1^{f 08013} / Y$ males ( $\mathrm{p}<0.01$, log-rank test) and with Actin5C-Gal4/+ males ( $\mathrm{p}<0.01$, log-rank test).

Furthermore, we investigated whether the overexpression of Ogg1 could confer resistance against paraquat on adult flies. Ogg $1^{f 01384} / Y$;Actin5C-Gal4/+ adult males showed a significantly longer lifespan even for diets containing $10 \mathrm{mM}$ paraquat, compared with $O g g 1^{f 01384} / Y$ males ( $\mathrm{p}<0.01$, log-rank test) and with Actin5C-Gal4/+ males ( $\mathrm{p}<0.01$, log-rank test) (Fig. 6C). We also obtained a similar result in experiments using another $P B a c\{W H\}$ allele, $O g g 1^{f 08013}$, although the observed lifespan extension under oxidative stress was less 

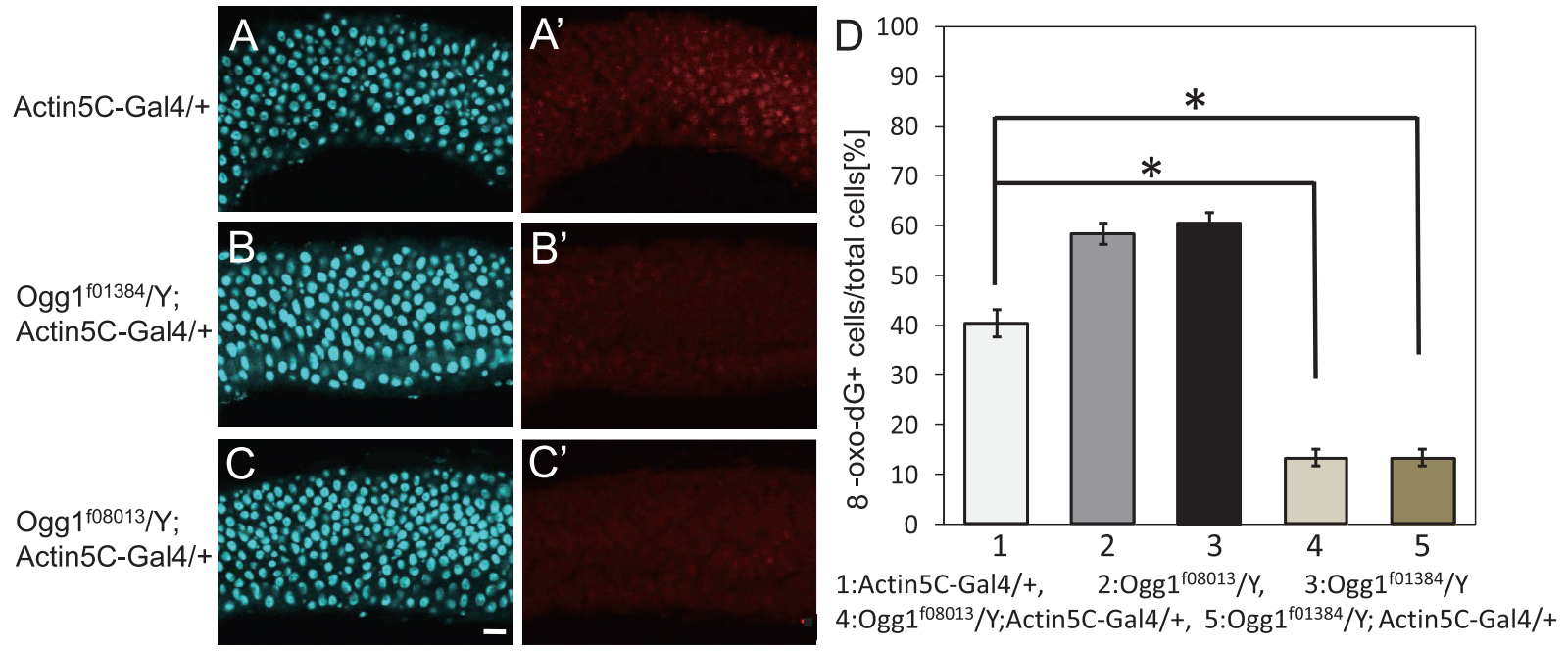

Fig. 5. Immunodetection of 8-oxo-guanines in posterior midgut cells from adults with Ogg1 overexpression after paraquat feeding. (A)-(C) Typical confocal images of epithelial cells from the posterior midgut region immunostained with anti-8-oxo-guanine antibody in control adult males and males with $O g g 1$ overexpression after $16 \mathrm{~h}$ of feeding on food containing $10 \mathrm{mM}$ paraquat. (A, A') Control epithelial midgut cells from an Actin5C-Gal4/+ male, stained with DAPI (A) and with anti-8-oxo-guanine antibody (A'). (B, B') Midgut cells from an adult fly with $O g g 1$ overexpression $\left(O g g 1^{f 01384} Y\right.$;Actin5C-Gal4/+), stained with DAPI (B) and with

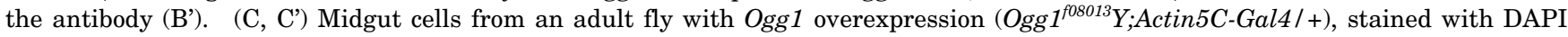
(C) and with the antibody (C'). (D) The frequencies of epithelial midgut cells stained with the antibody after paraquat feeding. Note that the frequency of midgut cells containing 8-oxo-guanines in their nuclei significantly decreased in adults with Ogg1 overexpression. Error bars represent the standard deviation. * ${ }^{*} \mathrm{p}<0.01$ (Student's $t$-test). Scale bar, $10 \mu \mathrm{m}$.
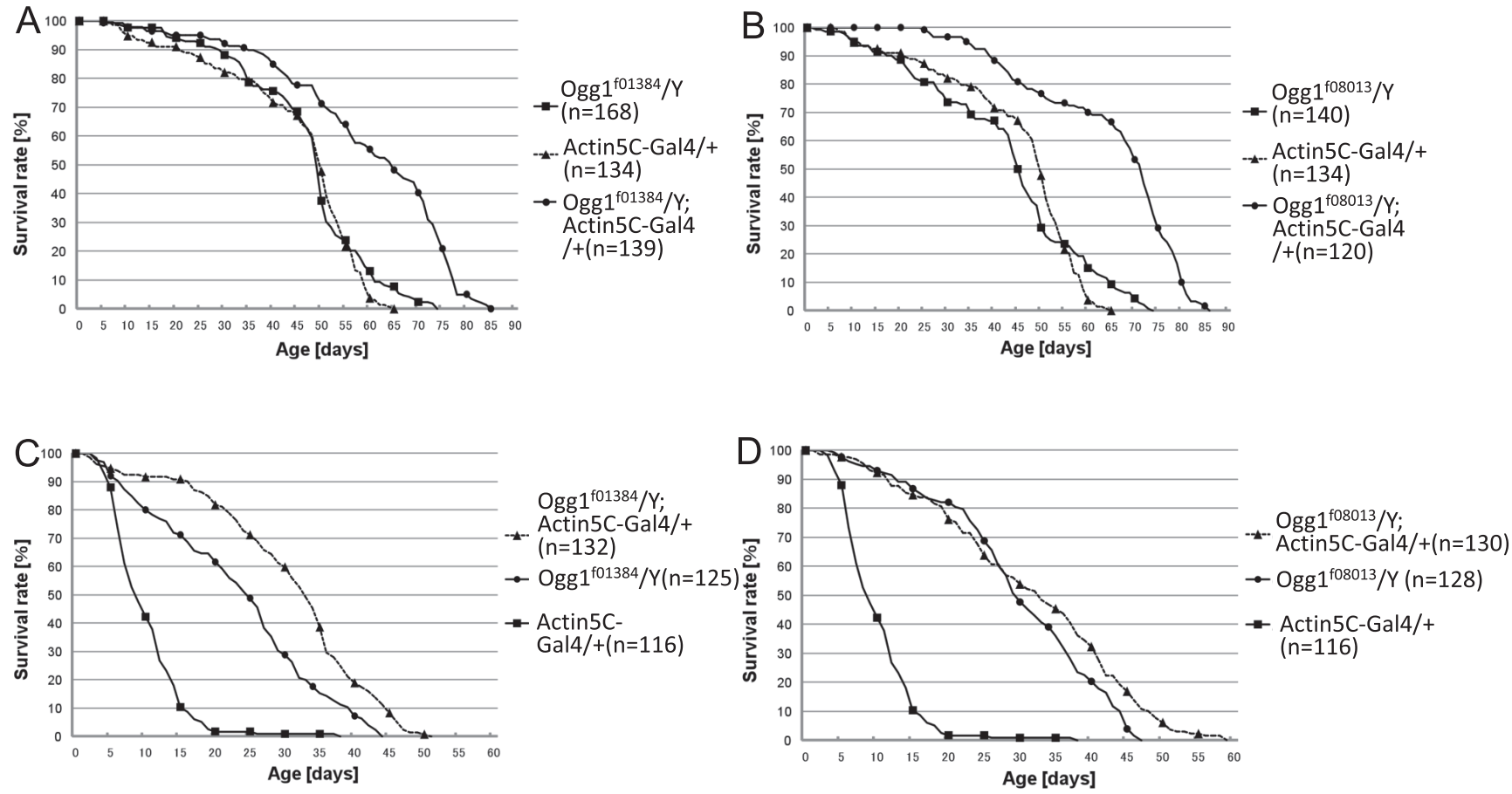

Fig. 6. Paraquat sensitivity assays and lifespan assays of adult males with Ogg1 overexpression. (A, B) Lifespan curves of adult males with $O g g 1$ overexpression in normal feeding conditions without paraquat. Ogg1 overexpression was performed in $O g g 1^{f 01384}$ /

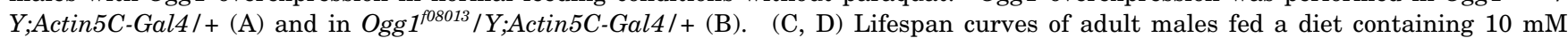
paraquat. Ogg1 overexpression was performed in Ogg1 $1^{f 01384} / Y$;Actin5C-Gal4/ + (C) and in Ogg $1^{\text {fosoli }} / Y$;Actin5C-Gal4/+ (D).

remarkable (Fig. 6D). However, the lifespan extension in $O g g 1^{f 08013} / Y$;Actin5C-Gal4/ + adult males was statistically significant, compared with Actin5C-Gal4/+ males
( $\mathrm{p}<0.05$, log-rank test) (Fig. 6D).

As described above, it is reasonable to conclude that the Drosophila Ogg1 ortholog is required for the suppression 
of 8-oxo-guanines generated by oxidative stress. Furthermore, we observed that adult flies with $\operatorname{Ogg} 1$ overexpression showed the acquisition of resistance to paraquat and lifespan extension in Drosophila adults. These phenotypes are probably a consequence of the efficient removal of 8-oxo-guanines in Drosophila adults, although we could not absolutely exclude the possibility that the lifespan extension and the paraquat resistance could result from a different genetic background. Several examples of gene overexpression resulting in lifespan extension in Drosophila adults have been reported. For example, overexpression of antioxidant enzymes such as SOD1, SOD2 and catalase caused lifespan extension (Orr and Sohal, 1994; Sun and Tower, 1999; Parkes et al., 1998; Sun et al., 2002). Overexpression of a Drosophila homolog of Sirtuin 2 (Sir2) (NAD+-dependent protein deacetylase) in fat bodies also led to an extended lifespan (Banerjee et al., 2012; Hoffmann et al., 2013). Although Sir2 orthologs are considered to be involved in the determination of lifespan in other organisms (Kaeberlein et al., 1999; Tissenbaum and Guarente, 2002), the ubiquitous overexpression of Drosophila Sir2 failed to extend the Drosophila adult lifespan (Burnett et al., 2011). However, its role in Drosophila lifespan determination should be reassessed. Overexpression of fatty-acid $\beta$ oxidationrelated genes can extend the lifespan of flies in a dietary restriction-related manner, mediated by an increased stress tolerance to oxidation and starvation (Lee et al., 2012). Moreover, overexpression of a protein repair methyltransferase that plays a role in restoring protein damage has an effect on lifespan (Chavous et al., 2001). However, it is still unknown whether overexpression of DNA repair enzymes would have a similar effect on adult lifespan in Drosophila. In this study, we first suggested that overexpression of a DNA glycosylase, Ogg1, resulted in hyper-resistance to oxidative stress and adult lifespan extension in Drosophila adults. ROS generated in the process of ATP production play a major role in oxidative DNA damage (Cadenas and Davies, 2000; Balaban et al., 2005). There is a well-known hypothesis that ROS contribute to the onset and development of many diseases such as obesity and diabetes, as well as aging (Harman, 1956, 1972; Pryor, 1987; Ames, 1989). It is possible to infer that the increased resistance to oxidative stress and the extended lifespan would be a consequence of enhanced activity to remove 8-oxo-guanines from mitochondrial and/or genomic DNA. Our results are consistent with the hypothesis that the accumulation of oxidative DNA damage is a primary cause of the senescence of organisms.

\section{CONCLUSIONS}

We showed in this study that Ogg1 mRNA levels decreased by $30-55 \%$ in Ogg1 mutants and in mRNA depletion experiments. In addition, we induced approximately 40-fold overexpression of the gene using the Gal4/ UAS system in Drosophila. Based on the positive correlation between the amounts of 8-oxo-guanines that accumulated and the level of Ogg1 expression, we could conclude that the Drosophila Ogg1 ortholog CG1795 plays an essential role in the suppression of oxidized guanine bases, consistent with previous results in other organisms. Adult flies with $\operatorname{Ogg} 1$ overexpression showed a resistance against oxidative stress by paraquat feeding and a significantly longer lifespan. These results are consistent with the hypothesis that oxidative DNA damage by ROS accumulation is a major contributor to senescence.

We would like to acknowledge Rie Awane, Kana Kaizuka and Ryotaro Okazaki for their technical assistance. We would also like to thank VDRC and BDSC for providing fly stocks. This study was partially supported by the JSPS Core-to-Core Program, B. Asia-Africa Science Platforms.

\section{REFERENCES}

Aburatani, H., Hippo, Y., Ishida, T., Takashima, R., Matsuba, C., Kodama, T., Takao, M., Yasui, A., Yamamoto, K., and Asano, M. (1997) Cloning and characterization of mammalian 8-hydroxyguanine-specific DNA glycosylase/apurinic, apyrimidinic lyase, a functional mutM homologue. Cancer Res. 57, 2151-2156.

Ames, B. N. (1989) Endogenous oxidative DNA damage, aging, and cancer. Free Radic. Res. Commun. 7, 121-128.

Arai, K., Morishita, K., Shinmura, K., Kohno, T., Kim, S. R., Nohmi, T., Taniwaki, M., Ohwada, S., and Yokota, J. (1997) Cloning of a human homolog of the yeast OGG1 gene that is involved in the repair of oxidative DNA damage. Oncogene 14, 2857-2861.

Audebert, M., Radicella, J. P., and Dizdaroglu, M. (2000) Effect of single mutations in the OGG1 gene found in human tumors on the substrate specificity of the Ogg1 protein. Nucleic Acids Res. 28, 2672-2678.

Balaban, R. S., Nemoto, S., and Finkel, T. (2005) Mitochondria, oxidants, and aging. Cell 120, 483-495.

Banerjee, K. K., Ayyub, C., Sengupta, S., and KolthurSeetharam, U. (2012) dSir2 deficiency in the fatbody, but not muscles, affects systemic insulin signaling, fat mobilization and starvation survival in flies. Aging (Albany NY) 4 , 206-223.

Barja, G. (2004) Free radicals and aging. Trends Neurosci. 27, 595-600.

Boiteux, S., and Radicella, J. P. (1999) Base excision repair of 8hydroxyguanine protects DNA from endogenous oxidative stress. Biochimie 81, 59-67.

Boiteux, S., and Radicella, J. P. (2000) The human OGG1 gene: structure, functions, and its implication in the process of carcinogenesis. Arch. Biochem. Biophys. 377, 1-8.

Burnett, C., Valentini, S., Cabreiro, F., Goss, M., Somogyvári, M., Piper, M. D., Hoddinott, M., Sutphin, G. L., Leko, V., McElwee, J. J., et al. (2011) Absence of effects of Sir2 overexpression on lifespan in C. elegans and Drosophila. Nature 477, 482-485.

Cadenas, E., and Davies, K. J. A. (2000) Mitochondrial free radical generation, oxidative stress, and aging. Free Radic. Biol. Med. 29, 222-230. 
Cappelli, E., D’Osualda, A., Bogliolo, M., Kelley, M. R., and Frosina, G. (2003) Drosophila S3 ribosomal protein accelerates repair of 8-oxoguanine performed by human and mouse cell extracts. Environ. Mol. Mutagen. 42, 50-58.

Chavous, D. A., Jackson, F. R., and O'Connor, C. M. (2001) Extension of the Drosophila lifespan by overexpression of a protein repair methyltransferase. Proc. Natl. Acad. Sci. USA 98, 14814-14818.

Chevillard, S., Radicella, J. P., Levalois, C., Lebeau, J., Poupon, M. F., Oudard, S., Dutrillaux, B., and Boiteux, S. (1998) Mutations in $O G G 1$, a gene involved in the repair of oxidative DNA damage, are found in human lung and kidney tumours. Oncogene 16, 3083-3086.

Cochemé, H. M., and Murphy, M. P. (2008) Complex I is the major site of mitochondrial superoxide production by paraquat. J. Biol. Chem. 283, 1786-1798.

Cooke, M. S., Evans, M. D., Dizdaroglu, M., and Lunec, J. (2003) Oxidative DNA damage: mechanisms, mutation, and disease. FASEB J. 17, 1195-1214.

Dherin, C., Dizdaroglu, M., Doerflinger, H., Boiteux, S., and Radicella, J. P. (2000) Repair of oxidative DNA damage in Drosophila melanogaster: identification and characterization of dOgg1, a second DNA glycosylase activity for 8hydroxyguanine and formamidopyrimidines. Nucleic Acids Res. 28, 4583-4592.

Girard, P. M., Guibourt, N., and Boiteux, S. (1997) The Ogg1 protein of Saccharomyces cerevisiae: a 7,8-dihydro-8-oxoguanine DNA glycosylase/AP lyase whose lysine 241 is a critical residue for catalytic activity. Nucleic Acids Res. 25, 32043211.

Grollman, A. P., and Moriya, M. (1993) Mutagenesis by 8oxoguanine: an enemy within. Trends Genet. 9, 246-249.

Harman, D. (1956) Aging: a theory based on free radical and radiation chemistry. J. Gerontol. 11, 298-300.

Harman, D. (1972) The biologic clock: the mitochondria? J. Am. Geriatr. Soc. 20, 145-147.

Hazra, T. K., and Mitra, S. (2006) Purification and characterization of NEIL1 and NEIL2, members of a distinct family of mammalian DNA glycosylases for repair of oxidized bases. Methods Enzymol. 408, 33-48.

Hoffmann, J., Romey, R., Fink, C., Yong, L., and Roeder, T. (2013) Overexpression of Sir2 in the adult fat body is sufficient to extend lifespan of male and female Drosophila. Aging (Albany NY) 5, 315-327.

Kaeberlein, M., McVey, M., and Guarente, L. (1999) The SIR2 / $3 / 4$ complex and SIR2 alone promote longevity in Saccharomyces cerevisiae by two different mechanisms. Genes Dev. $13,2570-2580$.

Klungland, A., Rosewell, I., Hollenbach, S., Larsen, E., Daly, G., Epe, B., Seeberg, E., Lindahl, T., and Barnes, D. E. (1999) Accumulation of premutagenic DNA lesions in mice defective in removal of oxidative base damage. Proc. Natl. Acad. Sci. USA 96, 13300-13305.

Kryston, T. B., Georgiev, A. B., Pissis, P., and Georgakilas, A. G. (2011) Role of oxidative stress and DNA damage in human carcinogenesis. Mutat. Res. 711, 193-201.

Lee, S. H., Lee, S. K., Paik, D., and Min, K. J. (2012) Overexpression of fatty-acid-beta-oxidation-related genes extends the lifespan of Drosophila melanogaster. Oxid. Med. Cell. Longev. 2012, 854502, 8 pages.

Lindahl, T. (1993) Instability and decay of the primary structure of DNA. Nature 362, 709-715.

Lu, R., Nash, H. M., and Verdine, G. L. (1997) A mammalian DNA repair enzyme that excises oxidatively damaged guanines maps to a locus frequently lost in lung cancer. Curr.
Biol. 7, 397-407.

Mao, G., Pan, X., Zhu, B. B., Zhang, Y., Yuan, F., Huang, J., Lovell, M. A., Lee, M. P., Markesbery, W. R., Li, G. M., et al. (2007) Identification and characterization of OGG1 mutations in patients with Alzheimer's disease. Nucleic Acids Res. 35, 2759-2766.

Maynard, S., Schurman, S. H., Harboe, C., de Souza-Pinto, N. C., and Bohr, V. A. (2009) Base excision repair of oxidative DNA damage and association with cancer and aging. Carcinogenesis 30, 2-10.

Michaels, M. L., Cruz, C., Grollman, A. P., and Miller, J. H. (1992) Evidence that $M u t Y$ and MutM combine to prevent mutations by an oxidatively damaged form of guanine in DNA. Proc. Natl. Acad. Sci. USA 89, 7022-7025.

Minowa, O., Arai, T., Hirano, M., Monden, Y., Nakai, S., Fukuda, M., Itoh, M., Takano, H., Hippou, Y., Aburatani, $\mathrm{H}$., et al. (2000) $M m h / O g g 1$ gene inactivation results in accumulation of 8-hydroxyguanine in mice. Proc. Natl. Acad. Sci. USA 97, 4156-4161.

Monden, Y., Arai, T., Asano, M., Ohtsuka, E., Aburatani, H., and Nishimura, S. (1999) Human MMH (OGG1) type 1a protein is a major enzyme for repair of 8-hydroxyguanine lesions in human cells. Biochem. Biophys. Res. Commun. 258, 605610.

Moriya, M. (1993) Single-stranded shuttle phagemid for mutagenesis studies in mammalian cells: 8-oxoguanine in DNA induces targeted G.C-->T.A transversions in simian kidney cells. Proc. Natl. Acad. Sci. USA 90, 1122-1126.

Nakabeppu, Y., Tsuchimoto, D., Yamaguchi, H., and Sakumi, K. (2007) Oxidative damage in nucleic acids and Parkinson's disease. J. Neurosci. Res. 85, 919-934.

Nash, H. M., Bruner, S. D., Scharer, O. D., Kawate, T., Addona, T. A., Spooner, E., Lane, W. S., and Verdine, G. L. (1996) Cloning of a yeast 8-oxoguanine DNA glycosylase reveals the existence of a base-excision DNA-repair protein superfamily. Curr. Biol. 6, 968-980.

Nemec, A. A., Wallace, S. S., and Sweasy, J. B. (2010) Variant base excision repair proteins: contributors to genomic instability. Semin. Cancer Biol. 20, 320-328.

Oka, S., Hirai, J., Yasukawa, T., Nakahara, Y., and Inoue, Y. H. (2015) A correlation of reactive oxygen species accumulation by depletion of superoxide dismutases with age-dependent impairment in the nervous system and muscles of Drosophila adults. Biogerontology. on line version DOI:10.1007/ s10522-015-9570-3

Okasaka, T., Matsuo, K., Suzuki, T., Ito, H., Hosono, S., Kawase, T., Watanabe, M., Yatabe, Y., Hida, T., Mitsudomi, T., et al. (2009) hOGG1 Ser326Cys polymorphism and risk of lung cancer by histological type. J. Hum. Genet. 54, 739-745.

Orr, W. C., and Sohal, R. S. (1994) Extension of life-span by overexpression of superoxide dismutase and catalase in Drosophila melanogaster. Science 263, 1128-1130.

Pandey, U. B., and Nichols, C. D. (2011) Human disease models in Drosophila melanogaster and the role of the fly in therapeutic drug discovery. Pharmacol. Rev. 63, 411-436.

Park, J. S., Lee, S. H., Na, H. J., Pyo, J. H., Kim, Y. S., and Yoo, M. A. (2012) Age- and oxidative stress-induced DNA damage in Drosophila intestinal stem cells as marked by GammaH2AX. Exp. Gerontol. 47, 401-405.

Parkes, T. L., Elia, A. J., Dickinson, D., Hilliker, A. J., Phillips, J. P., and Boulianne, G. L. (1998) Extension of Drosophila lifespan by overexpression of human SOD1 in motorneurons. Nat. Genet. 19, 171-174.

Paz-Elizur, T., Sevilya, Z., Leitner-Dagan, Y., Elinger, D., Roisman, L. C., and Livneh, Z. (2008) DNA repair of oxida- 
tive DNA damage in human carcinogenesis: potential application for cancer risk assessment and prevention. Cancer Lett. 266, 60-72.

Prüßing, K., Voigt, A., and Schulz, J. B. (2013) Drosophila melanogaster as a model organism for Alzheimer's disease. Mol. Neurodegener. 8, 35.

Pryor, W. A. (1987) The free-radical theory of aging revisited: a critique and a suggested disease-specific theory. In: Modern Biological Theories of Aging. (eds.: Warner, H. R., Butler, R. N., Sprott, R. L., and Schneider, E. L.), pp. 89112. Raven Press, New York.

Radicella, J. P., Dherin, C., Desmaze, C., Fox, M. S., and Boiteux, S. (1997) Cloning and characterization of hOGG1, a human homolog of the OGG1 gene of Saccharomyces cerevisiae. Proc. Natl. Acad. Sci. USA 94, 8010-8015.

Ravi, D., Wiles, A. M., Bhavani, S., Ruan, J., Leder, P., and Bishop, A. J. (2009) A network of conserved damage survival pathways revealed by a genomic RNAi screen. PLoS Genet. 5, e1000527.

Rosenquist, T. A., Zharkov, D. O., and Grollman, A. P. (1997) Cloning and characterization of a mammalian 8-oxoguanine DNA glycosylase. Proc. Natl. Acad. Sci. USA 94, 74297434.

Sakumi, K., Tominaga, Y., Furuichi, M., Xu, P., Tsuzuki, T., Sekiguchi, M., and Nakabeppu, Y. (2003) Ogg1 knockoutassociated lung tumorigenesis and its suppression by Mth1 gene disruption. Cancer Res. 63, 902-905.

Sampath, H., Vartanian, V., Rollins, M. R., Sakumi, K. Nakabeppu, Y., and Lloyd, R. S. (2012) 8-Oxoguanine DNA glycosylase (OGG1) deficiency increases susceptibility to obesity and metabolic dysfunction. PLoS One 7, e51697.

Sun, J., and Tower, J. (1999) FLP recombinase-mediated induction of $\mathrm{Cu} / \mathrm{Zn}$-superoxide dismutase transgene expression can extend the life span of adult Drosophila melanogaster flies. Mol. Cell. Biol. 19, 216-228.

Sun, J., Folk, D., Bradley, T. J., and Tower, J. (2002) Induced overexpression of mitochondrial $\mathrm{Mn}$-superoxide dismutase extends the life span of adult Drosophila melanogaster. Genetics 161, 661-672.

Thibault, S. T., Singer, M. A., Miyazaki, W. Y., Milash, B., Dompe, N. A., Singh, C. M., Buchholz, R., Demsky, M., Fawcett, R., Francis-Lang, H. L., et al. (2004) A complementary transposon tool kit for Drosophila melanogaster using $P$ and piggyBac. Nat. Genet. 36, 283-287.

Thomas, D., Scot, A. D., Barbey, R., Padula, M., and Boiteux, S. (1997) Inactivation of OGG1 increases the incidence of G. C--> T. A transversions in Saccharomyces cerevisiae: evidence for endogenous oxidative damage to DNA in eukaryotic cells. Mol. Gen. Genet. 254, 171-178.

Tissenbaum, H. A., and Guarente, L. (2002) Model organisms as a guide to mammalian aging. Dev. Cell 2, 9-19.

van der Kemp, P. A., Thomas, D., Barbey, R., de Oliveira, R., and Boiteux, S. (1996) Cloning and expression in Escherichia coli of the OGG1 gene of Saccharomyces cerevisiae, which codes for a DNA glycosylase that excises 7,8-dihydro-8-oxoguanine and 2,6-diamino-4-hydroxy-5-N-methylformamidopyrimidine. Proc. Natl. Acad. Sci. USA 93, 5197-5202.

Wallace, S. S. (1998) Enzymatic processing of radiation-induced free radical damage in DNA. Radiat. Res. 150, S60-S79.

Wang, D., Kreutzer, D. A., and Essigmann, J. M. (1998) Mutagenicity and repair of oxidative DNA damage: insights from studies using defined lesions. Mutat. Res. 400, 99-115.

Yacoub, A., Augeri, L., Kelley, M. R., Doetsch, P. W., and Deutsch, W. A. (1996) A Drosophila ribosomal protein contains 8-oxoguanine and abasic site DNA repair activities. EMBO J. 15, 2306-2312.

Yamaguchi, H., Kajitani, K., Dan, Y., Furuichi, M., Ohno, M., Sakumi, K., Kang, D., and Nakabeppu, Y. (2006) MTH1, an oxidized purine nucleoside triphosphatase, protects the dopamine neurons from oxidative damage in nucleic acids caused by 1-methyl-4-phenyl-1,2,3,6-tetrahydropyridine. Cell Death Differ. 13, 551-563. 